\title{
NUCLEAR RESONANCE FLUORESCENCE AND RAYLEIGH SCATTERING FROM ${ }^{203}$ TI
}

\author{
J. C. PALATHINGAL ${ }^{\dagger}$ and M. L. WIEDENBECK \\ Physics Department, University of Michigan, Ann Arbor, Michigan ${ }^{\dagger \dagger}$
}

Received 17 April 1967

\begin{abstract}
The lifetime of the $279 \mathrm{keV}$ excited state of ${ }^{203} \mathrm{Tl}$ was determined to be $0.28 \pm 0.04 \mathrm{nsec}$ by the method of nuclear resonance fluorescence, using the $212 \mathrm{keV}$ beta radiation for compensation of the recoil energy loss. Using gaseous and liquid sources and a thallium scatterer, the cross sections for resonance fluorescence and Rayleigh scattering processes were determined. The agreement between theory and experiment on Rayleigh scattering cross section obtained from the present measurement lends support to this value of lifetime.
\end{abstract}

NUCLEAR REACTION ${ }^{203} \mathrm{~T} l(\gamma, \gamma)$; measured $\sigma(\theta)$, resonance fluorescence. ${ }^{203} \mathrm{Tl}$ level deduced $T_{1}$.

\section{Introduction}

The lifetime of the $279 \mathrm{keV}$ state of ${ }^{203} \mathrm{Tl}$ has been the subject of several investigations by a number of different methods. In some of these cases, values of the lifetime have been quoted to a high degree of accuracy. It is, however, found that some of these values mutually disagree by several times the quoted error. We now report a value of the lifetime obtained as a result of a new measurement by a method not previously applied to this nuclear state. Two earlier reported values were obtained from determinations of nuclear resonance fluorescence scattering cross section by the centrifuge method ${ }^{1}$ ) and by the thermal method ${ }^{2}$ ). The present measurement is also based on nuclear resonance fluorescence, but the recoil compensation was obtained from the preceding $212 \mathrm{keV}$ beta emission (see fig. 1) using a gaseous source at a low pressure.

The cross sections of $279 \mathrm{keV}$ gamma rays for Rayleigh scattering and nuclear resonance fluorescence scattering from thallium were determined from the present experiment. A ratio of the two cross sections, independent of the source strength, electronic absorption of the gamma rays in the scatterer and the geometrical efficiency of the scatterer and the detector was also obtained. A check could therefore be made on the value of the resonance fluorescence scattering cross section by using this ratio and the theoretical expression for Rayleigh scattering cross section ${ }^{3-5}$ ). The latter has been approximately verified by previous experimental investigations ${ }^{6-8}$ ).

$\dagger$ Present address: Physics Department, University of Cincinnati, Cincinnati, Ohio.

t† Work supported by the U. S. Atomic Energy Commission. 


\section{Theoretical considerations}

The resonance absorption line of gamma rays usually has a width much larger than the natural line width $\Gamma$, owing to thermal motion of nuclei in the scatterer. In this case, the effective cross section for resonance fluorescence for a gamma ray of energy $E$ is given by ${ }^{9}$ )

$$
\sigma(E)=\frac{2 J_{1}+1}{2 J_{0}+1} \frac{1}{4} \lambda_{0}^{2} \frac{\Gamma_{0}}{\Gamma} \frac{\Gamma_{\mathrm{i}}}{\sqrt{\pi} \Delta} \exp \left\{-\left[\left(E-E_{\mathrm{r}}\right) / \Delta\right]^{2}\right\} .
$$

Here, $E_{\mathrm{r}}$ is the resonance energy and $J_{1}$ and $J_{0}$ the angular momenta of the nucleus in the excited and ground state, respectively. The constant $\lambda_{0}$ represents the wavelength of a gamma ray of energy equal to the energy of the nuclear excited state.

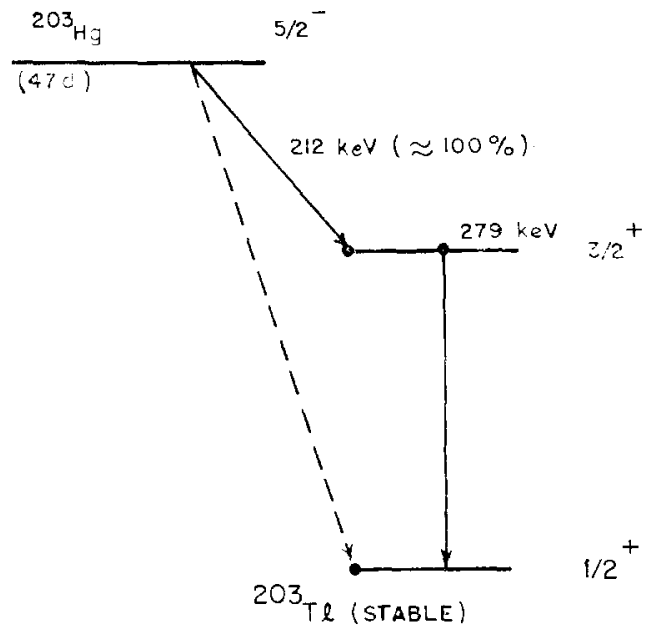

Fig. 1. Decay scheme of ${ }^{203} \mathrm{Hg}$ according to Nuclear Data Sheets (1962).

Similarly, $\Gamma_{0}$ stands for the partial energy level width for the direct gamma transition to the ground state and $\Gamma_{\mathrm{i}}$ the partial width for the particular mode of de-excitation of interest in a given measurement. The term $\Delta$ represents the Dopper width, which is the shift in the energy of the gamma ray when the nucleus travels in the direction of emission of the gamma quantum with a thermal kinetic energy equal to $k T$. It is given by

$$
\Delta=(E / c)(2 k T / M)^{\frac{1}{2}},
$$

where $M$ is the nuclear mass. For solid scatterers, $T$ used in eq. (2) is somewhat higher than the lab temperature ${ }^{9,10}$ ).

The energy of the gamma rays incident on the scatterer shall be given by

$$
E \approx E_{0}\left\{1-\frac{1}{M c}\left(p_{\beta} \cos \beta+p_{v} \cos y+\frac{E_{0}}{2 c}\right)+\frac{v_{\mathrm{th}}}{c}\right\},
$$




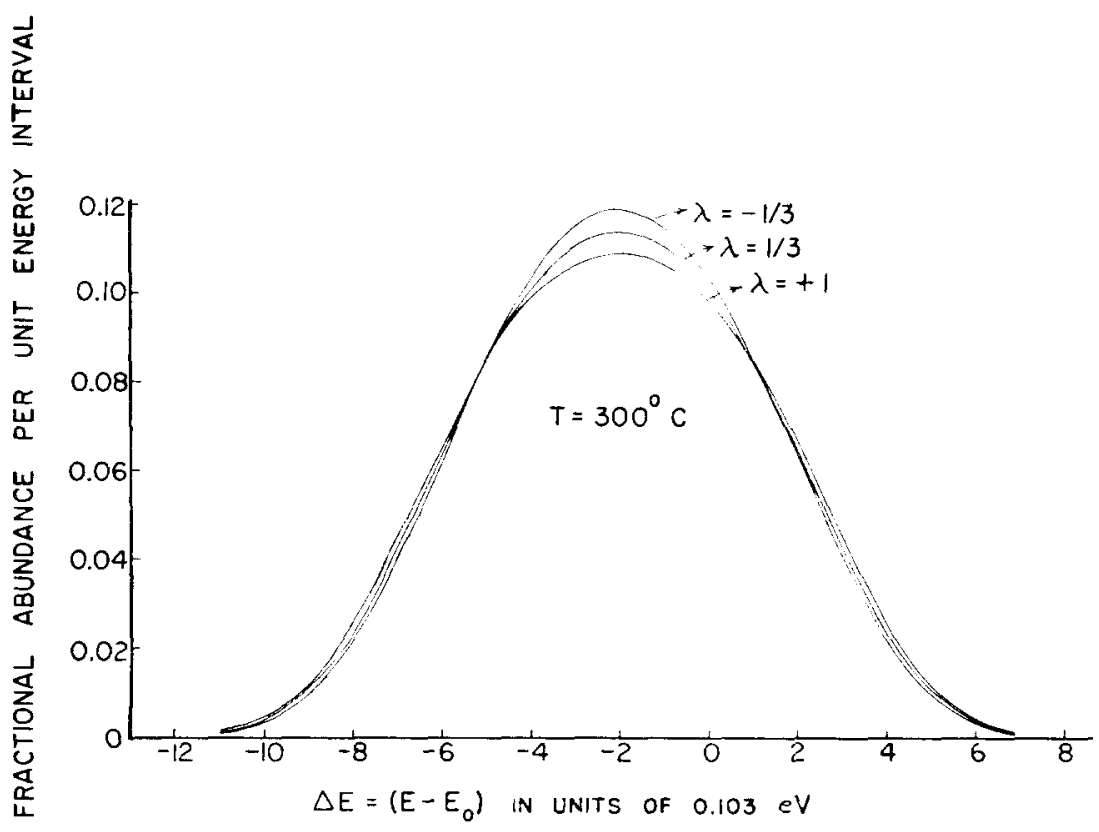

Fig. 2. Gamma-ray microspectra for different values of the beta-neutrino angular correlation coefficient $\lambda$, corresponding to $T=300^{\circ} \mathrm{C}$.

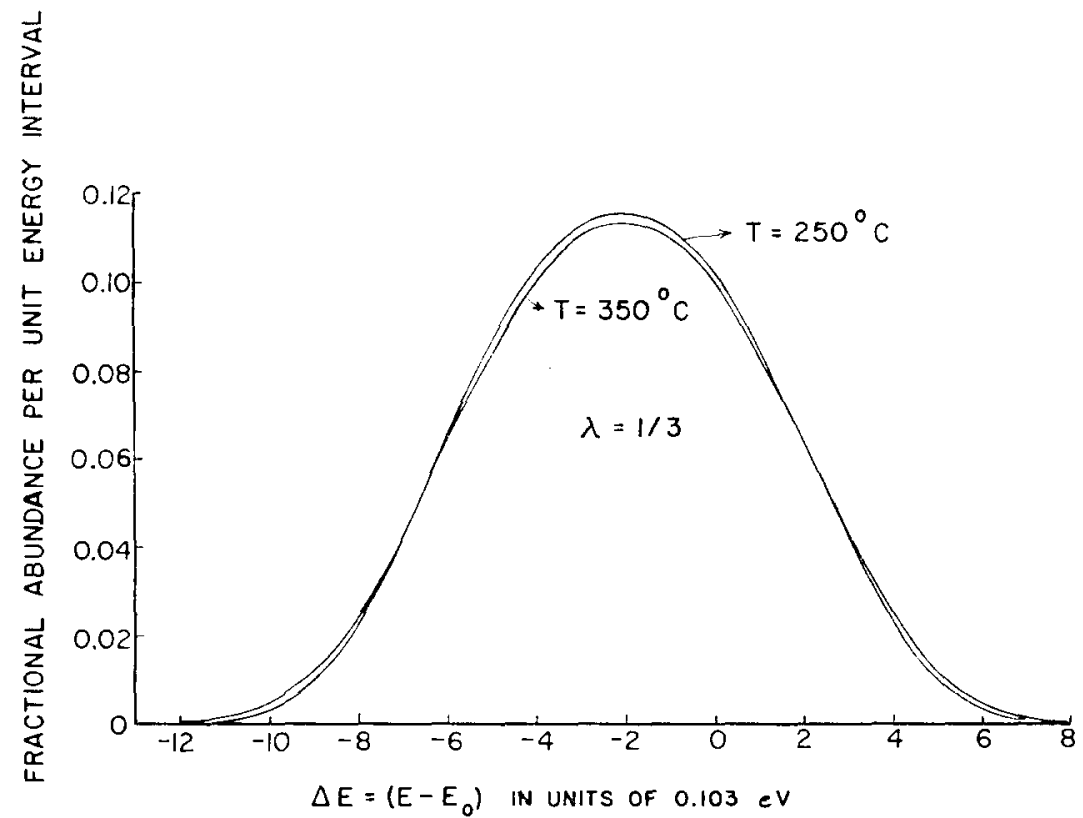

Fig. 3. Gamma-ray microspectra for two different temperatures for $\lambda_{2}=\frac{1}{1}-\frac{1}{3}$. 
where $p_{\beta}$ and $p_{v}$ represent the momenta of the electron and the neutrino, respectively, and $\beta$ and $v$ the angle between their respective directions and the direction of the gamma ray. The factor $v_{\text {th }}$ represents the component of the thermal velocity of the nucleus just before the beta emission, which is measured along the direction of the gamma ray. According to the different values of $p_{\beta}, p_{v}, \beta, v$ and $v_{\mathrm{th}}$, the gamma rays represent a continuous microspectrum. From the experimentally known shape of the beta-energy spectrum ${ }^{11}$ ), the gamma spectrum was calculated by a computer programme for different temperatures and for different beta-neutrino angular correlations. The beta-neutrino angular correlation is assumed to have the form

$$
W(\theta)=1+\lambda \frac{v}{c} \cos \theta
$$

with $\lambda$ taking values in the range +1 to $-\frac{1}{3}$. In the $\xi$-approximation which may be expected to be valid for ${ }^{203} \mathrm{Hg}$ beta decay ${ }^{12-13}$ ), such an angular correlation may be quite justified, as in the case ${ }^{14}$ ) of ${ }^{122} \mathrm{Sb}$. Illustrative results of the computations are given in figs. 2 and 3 , the former showing the variation for different $\lambda$-values at a source temperature of $300^{\circ} \mathrm{C}$ and the latter for temperatures $250^{\circ} \mathrm{C}$ and $350^{\circ} \mathrm{C}$, for $\lambda=+\frac{1}{3}$. It is found that the dependence on $\lambda$ or temperature is rather small. For a check on the results, the spectrum of gamma rays for a gaseous source of ${ }^{122} \mathrm{Sb}$ was also computed and found to agree with published results ${ }^{15}$ ).

Using eq. (1) and the computed shape of the incident gamma-ray spectrum, one may readily obtain the average resonance-scattering cross section $\sigma_{\text {res }}$ in terms of the line widths alone. An experimental determination of $\sigma_{\text {res }}$ therefore directly leads to a value of $\left(\Gamma_{\mathrm{o}} / \Gamma\right) \Gamma_{\mathrm{i}}$, and hence the mean life of the excited state.

\section{Experimental procedure}

The gaseous source was prepared by heating a small pellet of ${ }^{203} \mathrm{Hg}$ of $8.2 \mathrm{mg}$, enclosed in an evacuated and thoroughly cleaned quartz bulb of $6.5 \mathrm{~cm}^{3}$ volume. The ${ }^{203} \mathrm{Hg}$ had been obtained by irradiating the sealed high purity quartz (Suprasil, Engelhard Industries, Inc.) bulb containing ${ }^{202} \mathrm{Hg}$ (85\% enriched) in the Ford Research Reactor of the University of Michigan at a flux of $2.8 \times 10^{13}$ neutrons $\mathrm{cm}^{-2} \cdot \mathrm{sec}^{-1}$ for $122 \mathrm{~h}$.

Seven weeks after irradiation, no appreciable radioactivity was noticed except that of ${ }^{203} \mathrm{Hg}$. The specific activity of ${ }^{203} \mathrm{Hg}$ was expected to be about $0.26 \mathrm{mCi} / \mathrm{mg}$. The quartz bulb was placed inside a small Dewar flask and warmed so that no point inside the Dewar was at a temperature lower than $300^{\circ} \mathrm{C}$. At $280^{\circ} \mathrm{C}$, all the mercury was expected to go into the vapour state. The pressure inside the bulb was estimated to be $0.2 \mathrm{~atm}$. All the mercury was expected to exist in the form of monatomic molecules.

As shown in fig. 4 , the scatterer made of natural thallium $\left(29.5 \%{ }^{203} \mathrm{Tl}\right)$ in the form of a ring was placed symmetrically around the gamma detector, a $7.6 \mathrm{~cm} \times 7.6$ 
cm NaI (Tl) crystal coupled to a RCA 8054 photomultiplier. The energy resolution of the detector was $12 \%$ for $279 \mathrm{keV}$ gamma rays. The average gamma scattering angle was approximately $125^{\circ}$ and the minimum about $100^{\circ}$. Because of the large scattering angles, no substantial number of Compton gamma pulses or pile-up was registered in the full-energy region of the gamma spectrum.

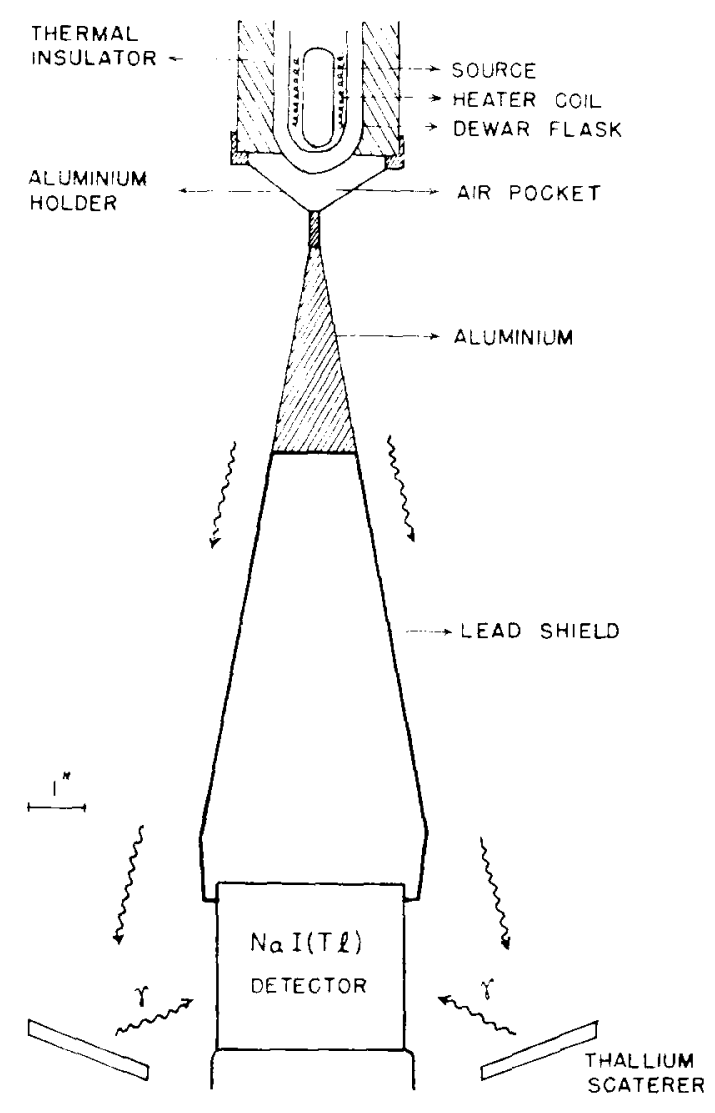

Fig. 4. A sketch of the apparatus.

The experiment was performed using the source in the gaseous and liquid states alternately. The mercury vapour was entirely converted into a liquid source of equal strength by simply cutting off power from the heater coil and allowing the source to cool to about the room temperature. The spectra of the scattered gamma rays for the liquid and gaseous sources were collected separately in different groups of 128 channels of a 512-channel, Nuclear Data analyser. Data were taken for two alternate runs of 80 live min each for either source, on each day for six days over a period of three weeks. 
Because of the high atomic number $(Z=81)$ of the scatterer and the fairly low energy of the gamma rays, a clear peak was obtained due to Rayleigh-scattered gamma rays when a liquid source was used (cross sections for other types of coherent scattering are negligible). A spectrum obtained using an aluminium scatterer contained no such peak. The peak seen in the case of thallium was about $10 \%$ higher for the gaseous source, evidently due to the superposition of resonance fluorescence scattering. A ratio of the Rayleigh and resonance scattering cross sections was readily obtained from the two types of spectra. The total area of each spectrum (after subtracting out the room background) was used as a normalization factor.

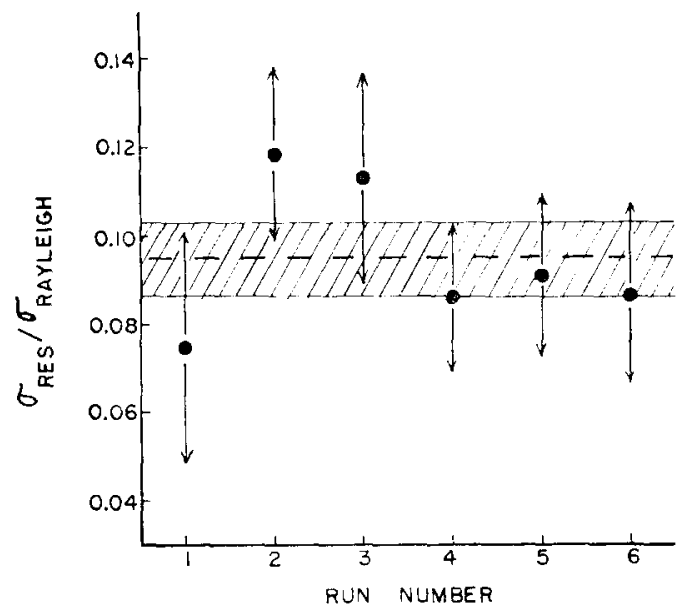

Fig. 5. Values of $\sigma_{\text {res }} / \sigma_{\text {Rayleigh }}$ obtained from the various runs and their weighted mean. The shaded area represents one standard deviation to either side of the mean value (the dashed line).

The ratio of the two cross sections shall yield the absolute value of either, if the absolute value of the other is determined. For this, however, one has to take into account the different angular distributions of the two types of coherently-scattered radiation. The differential Rayleigh scattering cross section for above is given according to the form factor approximation of Franz ${ }^{3,16}$ ) as

$$
\mathrm{d} \sigma(\theta) / \mathrm{d} \Omega=8.73\left(\frac{Z m c^{2}}{E}\right)^{3} \frac{\left(1+\cos ^{2} \theta\right)}{2 \sin ^{3} \frac{1}{2} \theta} \mathrm{nb} / \mathrm{sr} .
$$

This is remarkably constant over the range of scattering angle $\theta$ involved in this experiment. The angular distribution of the resonance gamma rays has been given to be ${ }^{1}$ )

$$
W(\theta)=1+(0.87 \pm 0.08) P_{2}(\cos \theta) .
$$

Since the angular distribution of the resonance radiation is quite anisotropic, and since the scatterer and the detector were placed very close to each other, it was 
necessary to average over the different gamma directions by a reasonably accurate empirical method. By this method, described below, the electronic absorption of the gamma rays scattered by Rayleigh or resonance processes from various layers of the scatterer was also determined. For this purpose, the scatterer was considered to be made of several volume elements. After removing the scatterer from the apparatus, a weak calibration source of known strength $S_{\text {cal }}$ relative to the experimental source was placed successively in positions corresponding to each of the volume elements and the photopeak singles counting rate separately determined. A second photopeak count rate was obtained for each position with a layer of lead placed just above the source parallel to the top surface of the scatterer facing the source. The thickness of the layer used in each case was such that the factor, electronic absorption coefficient $x$ thickness of the layer is equal to the corresponding factor for a layer of thallium of thickness $z$ equal to the depth of the test source position from the top surface of the scatterer. From the ratio of the two photopeak count rates, the average electronic absorption for gamma rays coherently scattered from each volume element of the scatterer was obtained. Since the gamma rays were incident almost normal to the top surface of the scatterer, this average absorption factor could be expressed as $\exp (\mu z \overline{\cos \theta})$, where $\mu$ is the absorption coefficient. It was therefore possible to obtain $\cos \theta$. Hence the approximate average angular distribution factor for radiation from each volume element was obtained using this value of $\overline{\cos \theta}$ in eq. (6). By weighing these factors according to the contribution to the resonance fluorescence counting rate from the corresponding volume element, the average angular distribution asymmetry factor $\overline{W(\theta)}$ for the entire scatterer could be obtained.

In determining the absolute values of the cross section, the weighted photopeak count rate when the calibration source was placed in positions corresponding to the various volume elements of the scatterer had to be known. This was readily available from the individual photopeak count rates obtained earlier. The ratio of this mean photopeak count rate $C_{\text {cal }}$ to the resonance fluorescence photopeak count rate $C_{\text {res }}$ shall be expressed as

$$
C_{\mathrm{res}} / C_{\mathrm{cal}}=S_{\mathrm{cal}}^{-1} \omega_{\mathrm{se}} N \sigma_{\mathrm{res}} f_{\mathrm{e}} \overline{W(\bar{\theta})}
$$

where $\omega_{\mathrm{sc}}$ is the geometrical efficiency of the scatterer, $N$ the number of nuclei per $\mathrm{cm}^{3}$ of ${ }^{203} \mathrm{Tl}$ atoms and $f_{\mathrm{e}}$ the empirically-obtained electronic transmission factor, the fraction of the resonance-scattered gamma rays that escape electronic absorption. It is significant that the value of $f_{\mathrm{e}}$ empirically determined by the method used here requires no correction for Compton gamma rays, scattered through small angles, that may not be distinguishable from coherently scattered radiation because the energy losses are too small to be measurable by the gamma detector of finite energy resolution. A relation similar to eq. (7) is valid for Rayleigh scattering. It may be noted, however, that all the atoms in the scatterer have to be taken into account for estimates on Rayleigh scattering. 


\section{Results and discussion}

The results on the ratio of the average resonance fluorescence scattering cross section to the average Rayleigh scattering cross section and their weighted mean are shown in fig. 5. The average value of differential Rayleigh scattering cross section for the present experimental geometry is estimated from eq. (5) to be $27.1 \mathrm{mb} / \mathrm{sr}$. The result of the present measurement $25.0 \pm 2.7 \mathrm{mb} / \mathrm{sr}$ is in agreement with this value. Based on this result and the mean value for the ratio of the

TABLE 1

Values of $\tau$ obtained by various observers

\begin{tabular}{clc}
\hline$\tau(\mathrm{nsec})$ & \multicolumn{1}{c}{ Method of measurement } & Ref. \\
\hline $0.28 \pm 0.04$ & $\begin{array}{l}\text { beta decay recoil } \\
\text { (resonance fluorescence) }\end{array}$ & present work \\
$0.408 \pm 0.010$ & delayed coincidence & 18 ) \\
$0.402 \pm 0.003$ & delayed coincidence & 19 ) \\
$0.410 \pm 0.020$ & centrifuge (resonance fluorescence) & ${ }^{1}$ ) \\
$0.405 \pm 0.008$ & delayed coincidence & ${ }^{20}$ ) \\
$0.348 \pm 0.014$ & delayed coincidence & ${ }^{21}$ ) \\
$0.408 \pm 0.025$ & delayed coincidence & ${ }^{22}$ ) \\
$0.41 \pm 0.03$ & delayed coincidence & ${ }^{23}$ ) \\
$0.32 \pm 0.04$ & high-frequency deflection & ${ }^{24}$ ) \\
$0.43 \pm 0.05$ & Coulomb excitation & ${ }^{25}$ ) \\
$0.42 \pm 0.04$ & delayed coincidence & ${ }^{26}$ ) \\
$0.38 \pm 0.07$ & thermal Doppler effect & \\
& (resonance fluorescence) & \\
$0.41 \pm 0.12$ & Coulomb excitation & ${ }^{27}$ ) \\
$0.18 \pm 0.05$ & delayed coincidence & ${ }^{28}$ ) \\
$0.3 \pm 0.1$ & delayed coincidence & ${ }^{29,}$ ) \\
$0.36 \pm 0.10$ & delayed coincidence &
\end{tabular}

Wherever a parameter other than $\tau$, but one directly related to it has been quoted as the result, $\tau$ is computed from this parameter.

two cross sections, the lifetime of the $279 \mathrm{keV}$ state of ${ }^{203} \mathrm{Tl}$ is found to be $\tau=$ $0.28 \pm 0.04 \mathrm{nsec}$. The internal conversion coefficient of the $279 \mathrm{keV}$ gamma rays is assumed to be 0.22 in this estimate ${ }^{17}$ ). The values of $\tau$ reported from the substantially large number of previous investigations are listed in table 1 . Our value is somewhat smaller than the majority of the quoted results, but it agrees with some. The good agreement between the theoretical and experimental values of the Rayleigh scattering cross section lends support to the value of the mean life, quoted here.

The authors are indebted to W. M. Greenberg for his valuable help in computing the microspectrum of the gamma rays. 


\section{References}

1) B. I. Deutch and F. R. Metzger, Phys. Rev. 122 (1961) 848

2) F. R. Metzger, J. Franklin Inst. 261 (1956) 219

3) W. Franz, Z. Phys. 95 (1935) 652, 98 (1935) 314

4) P. B. Moon, Proc. Phys. Soc. A63 (1950) 1189

5) G. E. Brown, R. E. Peierls and J. B. Woodward, Proc. Roy. Soc. A227 (1955) 51;

S. Brenner, G. E. Brown and J. B. Woodward, Proc. Roy. Soc. A227 (1955) 59;

G. E. Brown and D. F. Mayers, Proc. Roy Soc. A234 (1956) 387, A242 (1957) 89

6) A. Storruste and P. O. Tjom, Nuclear Physics 6 (1958) 151

7) A. M. Bernstein and A. K. Mann, Phys. Rev. 110 (1958) 805

8) K. G. Standing and J. V. Jovanovich, Can. J. Phys. 40 (1962) 622

9) F. R. Metzger in Progress in nuclear physics, Vol. 7, ed. by O. R. Frisch (Pergamon Press, New York, 1959) p. 58

10) W. E. Lamb, Phys. Rev. 55 (1939) 190

11) N. Marty, Compt. Rend. 240 (1955) 291

12) Z. Matumoto, M. Morita and M. Yamada, Bull. Kabayasi Inst. Phys. Res. 5 (1955) 210

13) T. Kotani and M. Ross, Phys. Rev. Lett. 1 (1958) 523

14) J. C. Palathingal, Phys. Rev. Lett. 14 (1965) 983

15) Y. K. Shubnyi, JETP (Sov. Phys.) 18 (1964) 316

16) C. M. Davisson, in Alpha-, beta- and gamma-ray spectroscopy, ed. by K. Siegbahn (NorthHolland Publ. Co., Amsterdam, 1965) p. 58

17) Nuclear Data Sheets (1962)

18) R. Rougny, J. J. Samueli and A. Sarazin, J. de Phys. 25 (1964) 989

19) Th. J. De Boer, H. Voorthuis and J. Blok, Physica 28 (1962) 417

20) A. Schwarzchild and J. Kane, Phys. Rev. 122 (1961) 854

21) E. C. B. Pederson and R. E. Bell, Nuclear Physics 21 (1960) 393

22) S. Gorodetzsky, R. Manquenouille, R. Richert and A. Knipper, Compt. Rend. 251 (1960) 65

23) E. Bashandy, T. R. Gerholm and J. Lindskog, Ark. Fys. 17 (1960) 421

24) B. Johansson and T. Alvager, Ark. Fys. 17 (1960) 163

25) F. K. McGowan and P. H. Stelson, Phys. Rev. 109 (1958) 901

26) E. E. Berlovich and G. V. Dubinkin, JETP (Sov. Phys.) 5 (1954) 164

27) R. Barloutaud, T. Grjebine and M. Riou, Physica 22 (1956) 1129A

28) H. de Waard, Phys. Rev. 99 (1955) 1045

29) R. E. Zauma and G. M. Lewis, Phil. Mag. 46 (1955) 1045

30) G. T. Ewan and R. L. Graham, unpublished 